\title{
L'exclusion du statut de réfugié est-elle compatible avec une protection effective des droits fondamentaux?
}

Is the Exclusion from Refugee Status Compatible with an Effective Protection of Fundamental Rights?

\section{Marina Eudes}

\section{(2) OpenEdition}

Journals

Édition électronique

URL : http://journals.openedition.org/crdf/1230

DOI : $10.4000 /$ crdf. 1230

ISSN : 2264-1246

\section{Éditeur}

Presses universitaires de Caen

\section{Édition imprimée}

Date de publication : 1 novembre 2015

Pagination : 103-111

ISBN : 978-2-84133-742-2

ISSN : $1634-8842$

\section{Référence électronique}

Marina Eudes, «L'exclusion du statut de réfugié est-elle compatible avec une protection effective des droits fondamentaux ? ", Cahiers de la recherche sur les droits fondamentaux [En ligne], 13 | 2015, mis en ligne le 01 novembre 2016, consulté le 11 février 2020. URL : http://journals.openedition.org/crdf/ 1230 ; DOI : 10.4000/crdf.1230 


\title{
L'exclusion du statut de réfugié est-elle compatible avec une protection effective des droits fondamentaux?
}

\author{
Marina EUDES \\ Maître de conférences (HDR) en droit public à l'université Paris Ouest Nanterre La Défense \\ Centre de recherches et d'études sur les droits fondamentaux (CREDOF, EA 3933) \\ Assesseur pour le Haut-Commissariat pour les réfugiés auprès de la Cour nationale du droit d'asile
}

I. L'appréciation du comportement susceptible de conduire à une exclusion à l'aune du principe de légalité

A. Une base légale existante mais pas uniformément interprétée

1. Un risque d'approches divergentes du crime international, du crime grave de droit commun ou des agissements contraires aux buts et principes des Nations unies

2. Une hypothèse d'atteinte à l'ordre public insuffisamment définie

B. Un manque de prévisibilité de la mise en jeu de la responsabilité

1. Une acception souple des divers modes de participation

2. La prise en compte variable de certaines causes d'exonération ou d'atténuation de responsabilité

II. Le nécessaire encadrement de la procédure d'adoption d'une décision d'exclusion

A. Des garanties procédurales élémentaires d'une particulière importance dans le contentieux de l'exclusion

1. L'impossibilité de déroger aux principes d'individualité et de confidentialité de l'examen de la demande 2. Les difficultés d'application du principe pourtant essentiel du contradictoire

B. Un standard de preuve élevé mais parfois mal respecté

1. Des appréhensions variables de la charge et du niveau de preuve requis en matière d'exclusion

2. La nécessité de réunir des éléments de preuve objectifs contre le demandeur pour l'exclure

«Une culture croissante de l'exclusion». Derrière ce titre accrocheur, des chercheurs présentaient en 2011 les grandes tendances du contentieux de l'exclusion du statut de réfugié tel qu'il ressortait de la jurisprudence des tribunaux canadiens entre 1998 et $2008^{1}$. Pour sortir d'un champ temporel et géographique nécessairement limité, des travaux similaires auraient pu être menés sur le continent européen pour, peut-être, aboutir à la même conclusion, à savoir l'impact négatif du contexte de la lutte contre le terrorisme international sur le taux national d'octroi du

1. A. Kaushal, C. Dauvergne, «The Growing Culture of Exclusion: Trends in Canadian Refugee Exclusions", International Journal of Refugee Law, vol. $23, \mathrm{n}^{\circ} 1,2011$, p. 54-92. 
statut de réfugié au sens de la Convention de $1951^{2}$. Or, la doctrine européenne n'a pas poursuivi une telle démarche, ce manque d'intérêt reflétant probablement le fait que les affaires d'exclusion sont encore très peu nombreuses ${ }^{3}$. Pourtant, si l'intrusion de considérations sécuritaires dans le contentieux de l'asile a toujours existé, elle a pris aujourd'hui une place probablement jamais atteinte auparavant, et ce, dans tous les États, notamment européens, attirant le plus grand nombre de demandeurs d'asile. De plus, le contentieux de l'exclusion, fondé sur l'article 1.F de la Convention de Genève ${ }^{4}$, devrait certainement se développer de manière significative tant d'un point de vue quantitatif que s'agissant des zones géographiques concernées. En effet, pendant longtemps, les cas d'exclusion apparaissant sur le continent européen, en particulier en France, concernaient essentiellement des ressortissants rwandais ou issus de l'ex-Yougoslavie; des personnes impliquées dans les divers crimes internationaux commis sur ces territoires dans les années 1990 avaient ensuite cherché à obtenir une protection internationale en Europe, mais s'étaient vu refuser celle-ci en raison de leur comportement passé. Aujourd'hui, avec la multiplication des conflits armés engendrant des violations massives des droits de l'homme et du droit international humanitaire, et par conséquent des afflux importants de demandeurs d'asile, il est probable que l'origine nationale des requérants se diversifie et que le nombre de cas d'exclusion progresse ${ }^{5}$. La survenue ou le prolongement des conflits armés en Syrie, au Soudan, en République centrafricaine ou encore en Irak, dans lesquels les différentes parties en présence peuvent participer à la commission d'exactions sur une grande échelle, entraîneront mathématiquement une augmentation du nombre d'affaires dans lesquelles la question de l'exclusion d'un demandeur d'asile pourra être posée ${ }^{6}$.
Dans ce contexte géopolitique également caractérisé par un durcissement général des politiques migratoires, il convient de déplorer le peu d'écrits interrogeant la place des droits fondamentaux dans le contentieux de l'exclusion$^{7}$. Cela est d'autant plus frappant que la doctrine est en revanche prolixe sur des sujets très proches de celui-ci, qu'il s'agisse des droits des demandeurs d'asile en général, de l'impact de la lutte contre le terrorisme sur l'effectivité des droits de l'homme ou encore des connexions entre droit de l'asile et droit international pénal ${ }^{8}$. Or il existe un lien consubstantiel unissant droit des réfugiés et droit des droits de l'homme, le premier corpus pouvant être considéré comme une lex specialis par rapport au second. Et cette optique devrait toujours être gardée à l'esprit malgré une conception unanime du mécanisme de l'exclusion comme une sanction justifiée par le souci de ne pas dévoyer le système de protection des réfugiés en en faisant bénéficier des personnes qui auraient elles-mêmes violé les droits d'autrui ${ }^{9}$. Tout comme la célèbre maxime «pas de liberté pour les ennemis de la liberté» trouve ses limites, les auteurs présumés d'exactions devraient pouvoir bénéficier de certains droits, malgré le jeu de la clause d'exclusion à leur égard. En pratique, cette affirmation semble toutefois peu effective vis-à-vis de certains demandeurs d'asile, en particulier ceux soupçonnés d'être impliqués dans la commission d'actes terroristes.

Au-delà des diverses questions éthiques que cela peut susciter ${ }^{10}, c^{\prime}$ est bien à une étude des aspects juridiques d'un paradoxe qu'il convient de s'atteler; celui de l'octroi de droits fondamentaux aux auteurs présumés de violations graves des droits de l'homme et du droit international humanitaire. Ce faisant, on pourra constater que malgré l'importance des standards liés au principe de légalité et des prises de position constantes du Haut-Commissariat

2. Convention du 28 juillet 1951 relative au statut des réfugiés (ci-après «Convention de Genève» ou «Convention de 1951 »).

3. Certains auteurs évaluent à moins d'un pour cent les demandes d'asile pouvant entraîner l'enclenchement de la logique de l'exclusion: J. Rikhof, The Criminal Refugee: The Treatment of Asylum Seekers with a Criminal Background in International and Domestic Law, Dordrecht, Republic of Letters, 2012, p. 369.

4. Art. 1.F de la Convention de Genève: «Les dispositions de cette Convention ne seront pas applicables aux personnes dont on aura des raisons sérieuses de penser: a) qu'elles ont commis un crime contre la paix, un crime de guerre ou un crime contre l'humanité, au sens des instruments internationaux élaborés pour prévoir des dispositions relatives à ces crimes; b) qu'elles ont commis un crime grave de droit commun en dehors du pays d'accueil avant d'y être admises comme réfugiés; c) qu'elles se sont rendues coupables d'agissements contraires aux buts et aux principesdes Nations Unies». On trouve des dispositions similaires dans la Convention régissant les aspects propres aux problèmes des réfugiés en Afrique, adoptée en 1969 par l'Organisation des États africains (ci-après "Convention de l'OEA »), le Statut du Haut-Commissariat pour les réfugiés (HCR) ou encore la directive 2011/95/UE du Parlement européen et du Conseil du 13 décembre 2011 concernant les normes relatives aux conditions que doivent remplir les ressortissants des pays tiers ou les apatrides pour pouvoir bénéficier d'une protection internationale, à un statut uniforme pour les réfugiés ou les personnes pouvant bénéficier de la protection subsidiaire, et au contenu de cette protection (ci-après «directive de 2011 " ou "directive "Qualification"»).

5. Dans le cas français, cette évolution est perceptible à la consultation des documents de la Cour nationale du droit d'asile (CNDA), en particulier le guide Trente ans de jurisprudence de la Cour nationale du droit d'asile et du Conseil d'État sur l'asile. Principales décisions de 1982 au 31 décembre 2011, et ses rapports annuels.

6. Dans le même sens, voir M. Kingsley Nyinah, «Exclusion under Art 1 F: Some Reflections on Context, Principles and Practice», International Journal of Refugee Law, vol. 12, suppl. 1, 2000, p. 301 sq.

7. Nous utilisons ici les notions de droits de l'homme et de droits fondamentaux comme synonymes, même si cette facilité de langage mériterait d'amples justifications.

8. Voir, en dernier lieu le dossier «The Interaction Between Refugee Law and International Criminal Justice» du Journal of International Criminal Justice, vol. 12, n ${ }^{\circ}$, numéro spécial, 2014.

9. Cette position était présente dès les travaux préparatoires de la Convention de Genève: voir J. Rikhof, The Criminal Refugee..., p. 51 sq. Voir aussi S. S. Juss, "Complicity, Exclusion and the "Unworthy" in Refugee Law», Refugee Survey Quarterly, vol. 31, n 3, 2012, p. 1-39.

10. Voir J. C. Simeon, "Ethics and the Exclusion of those Who Are "Not Deserving" of Convention Refugee Status", in Contemporary Issues in Refugee Law, S. S. Juss, C. J. Harvey (dir.), Cheltenham - Northampton, E. Elgar, 2013, p. 258-288. 
pour les réfugiés (HCR) ${ }^{11}$, les États abordent avec une grande latitude les actes d'un demandeur d'asile susceptibles d'entraîner son exclusion du statut de réfugié, entraînant ainsi un risque d'incohérence et de fragmentation de cette branche juridique (I). Il conviendra également de souligner la rareté des normes encadrant la mise en œuvre de ce mécanisme aux effets pourtant définitifs pour le candidat à l'asile (II).

\section{L'appréciation du comportement susceptible de conduire à une exclusion à l'aune du principe de légalité}

Même si l'on ne se trouve pas ici dans un contentieux pénal au sens strict, les conditions et conséquences de la mise en œuvre de la clause d'exclusion prévue à l'article 1.F de la Convention de Genève sont telles qu'elles doivent être lues à travers le prisme du principe de légalité ${ }^{12}$. La formulation même de l'article 1.F appelle d'ailleurs à une telle approche en ce qu'elle vise des «personnes dont on aura des raisons sérieuses de penser » qu'elles ont commis un «crime» les rendant indignes d'une protection internationale. Une analyse respectueuse des implications du principe de légalité ${ }^{13}$ suppose alors d'examiner avec beaucoup de circonspection le fondement de l'incrimination de l'acte reproché au demandeur d'asile. Ceci est d'autant plus vrai que le HCR rappelle constamment la nécessité d'interpréter étroitement la clause d'exclusion ${ }^{14}$, comme le sont traditionnellement les dispositions de limitation aux droits de l'homme. Or, l'appréciation du comportement passé d'un candidat à l'asile n'est pas toujours menée selon des critères clairs ni même homogènes selon les autorités nationales impliquées (A). On peut par ailleurs s'interroger sur la prévisibilité de la mise en jeu d'une responsabilité individuelle, alors même que les formes de participation à l'acte incriminé sont plurielles et que certains éléments peuvent mettre en cause la pertinence d'une décision d'exclusion (B).

\section{A. Une base légale existante mais pas uniformément interprétée}

Selon la Convention de Genève, les autorités nationales voulant appliquer la clause d'exclusion doivent avoir des raisons sérieuses de penser que le demandeur d'asile a commis un acte d'une telle gravité qu'il ne mérite pas de bénéficier de la protection internationale accordée aux réfugiés (1). Selon une disposition ajoutée par certaines législations nationales, et désormais uniformisée dans le cadre de l'Union européenne, l'exclusion peut également empêcher l'octroi de la protection subsidiaire en cas d'atteinte à l'ordre public de l'État sur le territoire duquel la demande d'asile est présentée (2).

\section{Un risque d'approches divergentes du crime international, du crime grave de droit commun ou des agissements contraires aux buts et principes des Nations unies}

La première hypothèse d'exclusion, envisagée par l'article 1.F.a de la Convention de 1951, est relativement aisée à identifier du fait de l'important développement de la jurisprudence nationale et internationale relative aux crimes de guerre, crimes contre l'humanité et génocide ${ }^{15}$. La codification du droit international pénal, initiée à la suite du procès de Nuremberg et illustrée dans les travaux de la Commission du droit international, certaines conventions, puis les statuts des tribunaux pénaux internationaux pour l'ex-Yougoslavie et le Rwanda ainsi que de la Cour pénale internationale, peut aussi guider aujourd'hui les autorités nationales responsables de l'octroi de l'asile ${ }^{16}$. Dépositaires du récit d'un demandeur, elles sont alors en mesure de confronter ses actes aux définitions des crimes internationaux précités et de l'exclure du statut de réfugié s'il est démontré qu'il a participé à ces exactions ${ }^{17}$.

L'article 1.F.b de la Convention de Genève évoque quant à lui l'exclusion liée à la participation d'un candidat à l'asile à la commission d'un «crime grave de droit

11. Le HCR a beaucoup travaillé sur ce thème de l'exclusion. Parmi les nombreux documents produits, il convient notamment de citer le chapitre IV du Guide des procédures et critères à appliquer pour déterminer le statut des réfugiés (décembre 2011, doc. HCR/1P/4/FRE/REV.3), les Principes directeurs sur la protection internationale $n^{\circ} 5$. Application des clauses d'exclusion (4 septembre 2003, doc. HCR/GIP/O3/05) et la Note d'information sur l'application des clauses d'exclusion: article $1 \mathrm{~F}$ de la Convention de 1951 relative au statut des réfugiés (4 septembre 2003).

12. Le principe de légalité, présent dans tous les textes internationaux relatifs aux droits de l'homme et dans le système juridique de tout État de droit, implique que la poursuite et la condamnation d'un individu reposent sur une base légale, c'est-à-dire que l'acte qu'on lui reproche devait être criminalisé au moment de sa commission. Le principe de légalité est également conçu comme supposant l'accessibilité de la norme prohibant certains comportements, et la prévisibilité de la mise en jeu de la responsabilité pénale individuelle quand de tels actes sont commis.

13. Selon le Conseil constitutionnel, «la gravité du crime susceptible d'exclure une personne [...] ne peut être appréciée qu'à la lumière des principes du droit pénal français" (CC, déc. $\mathrm{n}^{\circ} 2003-485$ DC du 4 décembre 2003, Loi modifiant la loi $n^{\circ} 52-893$ du 25 juillet 1952 relative au droit d'asile; nous soulignons).

14. Voir notamment les Principes directeurs sur la protection internationale $n^{\circ} 5 \ldots, \$ 2$.

15. Cette disposition a été pensée dans le contexte de l'après-Seconde Guerre mondiale, notamment marqué par la mise en place du tribunal militaire international de Nuremberg, et le jugement des grands criminels nazis. Elle contient ainsi une référence aux instruments internationaux relatifs à ce type de crimes.

16. Il faudrait d'ailleurs distinguer la portée de ces différentes sources sur l'office des instances nationale d'asile, comme le souligne C. Bauloz dans son article "L'apport du droit international pénal au droit des réfugiés», in Asile et extradition: théorie et pratique de l'exclusion du statut de réfugié, V. Chetail, C. Laly-Chevalier (dir.), Bruxelles, Bruylant, 2014, p. 35 sq.

17. Une brève définition des crimes contre la paix, crimes de guerre et crimes contre l'humanité est notamment proposée par le HCR dans ses Principes directeurs sur la protection internationale $n^{\circ} 5 \ldots, \S 11-13$. De très nombreux exemples jurisprudentiels nationaux sont étudiés dans J. Rikhof, The Criminal Refugee..., p. 124 sq. 
commun » ou un «crime non politique» (pour reprendre la terminologie éclairante de la version anglaise du texte et de la Convention de l'OEA). L'objectif est ici d'exclure de la protection internationale les individus impliqués dans la commission de graves actes criminels (qui peuvent $\mathrm{du}$ reste justifier l'enclenchement de procédures d'extradition $)^{18}$ et dont la présence constituerait une menace pour la sécurité des États d'accueil ${ }^{19}$. La principale application de cette logique concerne les personnes suspectées de participer à des activités terroristes ${ }^{20}$, mais on trouve dans la jurisprudence de nombreux autres actes commis avec violence $^{21}$, l'appréciation de la gravité se faisant au regard de divers critères tels que la nature des comportements en cause et des dommages qu'ils ont causés ou encore le quantum des peines encourues.

La troisième circonstance justifiant une exclusion, prévue à l'article 1.F.c de la Convention de 1951, repose sur la participation du demandeur d'asile à des «agissements contraires aux buts et principes des Nations Unies». S'interrogeant sur le sens de cette formule et sa réception en jurisprudence, la doctrine a considéré qu'il s'agissait ici essentiellement de viser des attaques menées contre les personnels onusiens, des cas de violations graves des droits de l'homme ou encore des faits de terrorisme ${ }^{22}$. Avec ces deux dernières illustrations, on perçoit la possibilité de recoupement avec les hypothèses précédemment étudiées de crimes contre l'humanité ou de crimes graves de droit commun, ce qui brouille évidemment la classification envisagée par les rédacteurs de la Convention de Genève ${ }^{23}$.

Alors qu'une certaine uniformité des jurisprudences est souhaitable en la matière ${ }^{24}$, des divergences d'interprétation apparaissent ponctuellement entre États, voire au sein d'un même État; une même catégorie d'actes peut ainsi aboutir ou non à une décision d'exclusion, laquelle peut elle-même se fonder sur l'un ou l'autre alinéa de l'article 1.F de la Convention de $1951^{25}$. S'agissant de crimes a priori caractérisés par leur gravité, de tels errements sont évidemment regrettables et interrogent sur l'effectivité du principe de légalité des délits et des peines voulant qu'un même acte entraîne les mêmes conséquences juridiques. Sans un suivi régulier et attentif du droit comparé de l'asile et du droit international pénal par les instances nationales, c'est alors une problématique fragmentation du droit des réfugiés qui pourrait apparaître, solution déjà dénoncée s'agissant de certains pays ${ }^{26}$.

Ce décalage ponctuel entre droit international et systèmes juridiques internes est du reste perceptible dans un autre domaine de mise en œuvre de la logique de l'exclusion.

\section{Une hypothèse d'atteinte à l'ordre public insuffisamment définie}

Parallèlement au système prévu par la Convention de Genève, certains États ont choisi d'attribuer une protection subsidiaire aux candidats à l'asile ne répondant pas aux conditions d'obtention du statut de réfugié, mais pour lesquels il existe des motifs sérieux et avérés de croire qu'ils courraient dans leur pays un risque réel de subir la peine de mort, la torture ou des peines ou traitements inhumains ou dégradants ou encore, pour des civils, une menace grave et individuelle en raison d'une violence aveugle résultant d'une situation de conflit armé ${ }^{27}$. Dans un légitime souci d'unification des législations et pratiques adoptées sur le continent européen, la directive «Qualification» a prévu pour cette protection subsidiaire qu'elle autorise les mêmes causes d'exclusion que pour la protection conventionnelle classique ${ }^{28}$. Elle a toutefois ajouté une clause d'ordre public permettant de ne pas octroyer ladite protection à un individu représentant «une menace pour la société ou la sécurité de l'État membre dans lequel il se trouve ${ }^{29}$. Pensée dans le contexte de la lutte internationale contre

18. V. Chetail a bien montré les points de différence mais aussi de convergence entre ces deux branches juridiques: «Les relations entre le droit de l'extradition et le droit des réfugiés: étude de l'article $1 \mathrm{~F}(\mathrm{~B})$ de la Convention de Genève du 28 juillet 1951", in Asile et extradition..., p. 65 -91.

19. Le crime reproché au demandeur d'asile doit toutefois avoir été commis à l'étranger, et non sur le territoire de l'État d'accueil: voir notamment CE, 25 septembre 1998, M. R., $\mathrm{n}^{\circ} 165525$.

20. HCR, Principes directeurs sur la protection internationale $n^{\circ} 5 \ldots, \S 26$.

21. Voir les exemples cités par V. Chetail, «Les relations entre le droit de l'extradition et le droit des réfugiés... », p. 70-71.

22. Voir S. Sivakumaran, «Exclusion from Refugee Status: The Purposes and Principles of the United Nations and Article 1 F(c) of the Refugee Convention », International Journal of Refugee Law, vol. 26, n³ 3, 2014, p. 350-381; P. d'Argent, P. d'Huart, "L'article 1 F c de la Convention de Genève relative au statut de réfugié du 28 juillet 1951: éléments matériels», in Asile et extradition..., p. 93-120.

23. Les Principes directeurs sur la protection internationale $n^{\circ} 5 \ldots$ du HCR ne répondent que partiellement à cette confusion en limitant l'applicabilité de l'article 1.F.c à des «circonstances extrêmes", à l'égard d'activités mettant en cause «le fondement même de la coexistence de la communauté internationale», $\$ 17$.

24. Plusieurs auteurs font d'ailleurs le constat des points de convergences nombreux entre certains États. C'est notamment le cas dans la thèse de M. Tissier-Raffin consacrée aux jurisprudences de l'Australie, de la Belgique, du Canada, des États-Unis, de la France, de la Grande-Bretagne et de la Nouvelle-Zélande, La qualité de réfugié de l'article 1 de la Convention de Genève à la lumière des jurisprudences occidentales, thèse de doctorat en droit public, sous la direction de S. Szurek, université Paris Ouest Nanterre La Défense, 2013, p. 128-178 et dans l'ouvrage de J. Rikhof, The Criminal Refugee..., p. 369-377.

25. Voir les conclusions des articles précités de l'ouvrage de V. Chetail et C. Laly-Chevalier, Asile et extradition...

26. Ainsi, aux États-Unis, l'absence de toute référence aux textes internationaux dans la législation fédérale pertinente aboutit à un système éloigné de ce qui devrait découler de la Convention de Genève: voir K. Jastram, «Left Out of Exclusion. International Criminal Law and the "Persecutor Bar" in US Refugee Law», Journal of International Criminal Justice, vol. 12, nº 5, 2014, p. 1183-1199.

27. En France, la protection subsidiaire est envisagée à l'article L. 712-2 du Code de l'entrée et du séjour des étrangers et du droit d'asile (CESEDA).

28. Si ce n'est qu'elle précise que la clause d'exclusion visant les auteurs de crimes graves concerne aussi les actes commis dans le pays de refuge, pas seulement ceux commis à l'étranger, ce qui est une extension regrettable par rapport aux dispositions de la Convention de Genève.

29. Art. 17, §1, d, de la directive «Qualification». 
le terrorisme, cette clause s'avère problématique en ce que les formules qu'elle utilise ne sont pas définies, laissant aux autorités nationales une grande latitude pour apprécier ce qui relève de ces menaces pour la société et la sécurité de l'État ${ }^{30}$. De plus, cette clause d'ordre public entretient une certaine confusion avec le cas du réfugié statutaire dont le refoulement est décidé au motif qu'il représente une menace pour la sécurité ou la communauté du pays d'accueil ${ }^{31}$. Outre le manque de lisibilité que cela entraîne, on constate un changement de position du curseur, l'objectif de protection jouant finalement moins au profit des individus qu'au bénéfice des États, contrairement à la volonté des rédacteurs de la Convention de $1951^{32}$.

Qu'elle vise uniquement la protection subsidiaire ou qu'elle soit étendue à la protection conventionnelle (comme le souhaitent certains États), la clause d'ordre public constitue à nouveau un facteur de fragmentation du droit des réfugiés en ce qu'elle permet aux instances nationales d'adopter des appréciations variables des actes susceptibles de justifier une exclusion. Or, une telle décision est par ailleurs difficilement prévisible s'agissant du degré de participation exigé à ces actes et du fait de la prise en compte éventuelle de certains éléments propres au candidat à l'asile.

\section{B. Un manque de prévisibilité de la mise en jeu de la responsabilité}

Si l'exclusion des personnes ayant participé à la commission des actes visés à l'article 1.F de la Convention de Genève semble légitime, force est de constater que le degré de participation exigé est entendu de manière très large, voire divergente selon le pays dans lequel la demande d'asile est présentée (1). Certaines circonstances propres au demandeur peuvent par ailleurs remettre en cause la logique de l'exclusion (2).

\section{Une acception souple des divers modes de participation}

Si la directive de 2011 apporte un intéressant éclaircissement par rapport à la Convention de 1951 en soulignant que la clause d'exclusion «s'applique aux personnes qui sont les instigatrices des crimes ou des actes [litigieux], ou qui y participent de quelque autre manière ${ }^{33}$, un certain flou caractérise en pratique l'acception du degré de participation exigé. Au-delà des cas dans lesquels il serait démontré que le demandeur d'asile, auteur principal, a lui-même commis ou ordonné le crime litigieux, c'est en réalité une multitude de circonstances qui peuvent être retenues contre lui et justifier son exclusion du statut de réfugié. Le HCR admet cette solution mais ne détaille pas les conditions à suivre en la matière, se contentant d'énoncer qu'il n'est pas nécessaire «que la personne ait physiquement commis l'acte criminel en question. L'instigation, la complicité et la participation à une entreprise criminelle commune peuvent suffire ${ }^{34}$. Parmi ces modes de participation, les autorités nationales privilégient souvent la complicité, généralement plus aisée à manier. Ce faisant, elles n’ont pas toujours suivi l'interprétation de cette notion proposée par les juridictions pénales internationales. Sans revenir ici sur le détail des jurisprudences pertinentes ${ }^{35}$, on peut regretter que le lien entre le comportement de l'individu et le crime dont on le pense complice soit parfois ténu; un même acte pouvant donc entraîner ou non la mise en ouvre de la clause d'exclusion selon l'instance qui l'examinera ${ }^{36}$. Dans ce contexte d'appréciation souple des modes de participation admissibles, il convient d'insister sur l'importance d'appliquer un standard élevé de preuve de l'implication concrète et personnelle du demandeur d'asile dans la commission du crime ${ }^{37}$.

\section{La prise en compte variable de certaines causes d'exonération ou d'atténuation de responsabilité}

Certains éléments objectifs peuvent aboutir à une mise à l'écart de la responsabilité d'un demandeur d'asile et donc de la clause d'exclusion à son égard. La circonstance la plus aisée à saisir est celle de la minorité de l'intéressé au moment de la commission des faits qui auraient pu entraîner son exclusion du statut de réfugié. Cette solution semble relativement bien admise, car fondée sur le principe de majorité pénale tel que conçu en droit international pénal ou en droit des droits de l'homme, ainsi que sur

30. En France, le texte pertinent évoque « une menace grave pour l'ordre public, la sécurité publique ou la sûreté de l'État». Plus généralement sur cet article L. 712-2, d, du CESEDA, voir F. Boggio-Cosadia, «Protection subsidiaire et menace à l'ordre public», in Asile et extradition..., p. 121-142.

31. L'article $33, \$ 2$, de la Convention de Genève vise « un réfugié qu'il y aura des raisons sérieuses de considérer comme un danger pour la sécurité du pays où il se trouve ou qui, ayant été l'objet d'une condamnation définitive pour un crime ou délit particulièrement grave, constitue une menace pour la communauté dudit pays".

32. De ce fait, une certaine réserve s'impose à l'égard de la proposition d'amendement au projet de loi français relatif à la réforme de l'asile visant à étendre l'applicabilité de la clause d'ordre public à la protection conventionnelle elle-même: amendement proposé par le rapporteur M. Buffet à la commission des lois du Sénat le 17 avril 2015 (doc. COM-242 rect.).

33. Art. 12, \$3, de la directive "Qualification».

34. HCR, Principes directeurs sur la protection internationale $n^{\circ} 5 \ldots, \$ 18$.

35. Pour s'en tenir au cas français, le complice est entendu comme «celui qui, sciemment ou par ses agissements, a contribué à la préparation ou à la réalisation du crime ou en a facilité la commission ou a assisté à son exécution sans chercher à aucun moment, eu égard à sa situation, à le prévenir ou à s'en dissocier» (CE, 26 janvier 2011, M. H., nº 312833).

36. Voir C. Bauloz, «L’apport du droit international pénal...», p. $42 s q$

37. Voir développements du II.B infra. 
la perception généralement partagée de la particulière vulnérabilité caractérisant les mineurs ${ }^{38}$. En dehors de ce cas précis, il convient de constater des divergences dans l'admission des autres causes envisageables d'atténuation ou d'exonération de responsabilité. Ainsi en est-il particulièrement de l'expiation des demandeurs d'asile impliqués dans la commission d'actes relevant de la logique de l'exclusion: si le HCR et certaines autorités nationales admettent que l'exécution d'une peine et le repentir d'un ancien criminel peuvent suffire à ce que le statut de réfugié lui soit accordé en cas de craintes de persécution ${ }^{39}$, d'autres refusent catégoriquement une telle solution ${ }^{40}$. Les autres causes possibles d'exonération ou d'atténuation de la responsabilité, en particulier la légitime défense, l'ordre du supérieur, la contrainte ou l'état de nécessité ne semblent quant à elles pas avoir fait l'objet de suffisamment d'applications jurisprudentielles pour que des règles précises les concernant puissent être dégagées, si ce n'est un principe d'interprétation stricte ${ }^{41}$.

Finalement, l'examen des éléments retenus pour apprécier le comportement d'un demandeur d'asile susceptible de conduire à son exclusion démontre le caractère casuistique, voire aléatoire, de ce contentieux. Dans ce contexte de relative mise à mal du principe de légalité, il est impératif de vérifier qu'une compensation s'opère par le biais de la mise en œuvre effective de certains principes visant à encadrer la procédure en cause.

\section{Le nécessaire encadrement de la procédure d'adoption d'une décision d'exclusion}

Le premier élément pouvant être évoqué comme gage de protection des droits du demandeur d'asile susceptible de tomber sous le coup d'une clause d'exclusion repose sur le respect de l'ordre «inclusion-exclusion» dans le traitement de sa demande ${ }^{42}$. Cela signifie que doivent d'abord être examinées ses craintes de persécution au sens de la Convention de Genève, et ensuite, si elles sont fondées, les causes possibles d'exclusion; ceci afin de faire jouer, même en cas d'exclusion, le principe de nonrenvoi de l'intéressé vers son pays d'origine s'il risque d'y être soumis à des mauvais traitements ${ }^{43}$. En outre, le demandeur d'asile potentiellement visé par la clause d'exclusion doit bénéficier des garanties offertes de façon générale à tout candidat à l'asile ${ }^{44}$, en particulier le droit de bénéficier d'une procédure individualisée, confidentielle et contradictoire (A). Il doit également profiter d'un certain niveau d'exigence en termes d'administration de la preuve, du fait de la gravité des actes qui peuvent lui être imputés et des conséquences qui en seront tirées (B).

\section{A. Des garanties procédurales élémentaires d'une particulière importance dans le contentieux de l'exclusion}

Outre un examen individuel et confidentiel de sa demande (1), le candidat à l'asile à qui les autorités envisagent d'opposer une clause d'exclusion doit bénéficier du principe du contradictoire (2). Si ces garanties ne sont pas spécifiques au contentieux de l'exclusion, elles présentent ici une importance particulière, s'agissant d'un demandeur qui pourra être jugé indigne d'une protection: cette indignité potentielle qui pourrait l'empêcher d'accéder au statut de réfugié ou à la protection subsidiaire ne peut en effet justifier à elle seule une mise à l'écart de certains principes fondamentaux ${ }^{45}$.

\section{L'impossibilité de déroger aux principes d'individualité et de confidentialité de l'examen de la demande}

Toute demande d'asile doit être instruite individuellement ${ }^{46}$. Si ce principe se justifie par la nécessité de prendre du temps pour appréhender la complexité de chaque

38. HCR, Principes directeurs sur la protection internationale $n^{\circ} 5 \ldots, \$ 28$ et Note d'information sur l'application des clauses d'exclusion..., $\$ 91-93$. Voir aussi J. Rikhof, The Criminal Refugee..., p. 287-295 au sujet des enfants soldats.

39. Voir notamment HCR, Principes directeurs sur la protection internationale $n^{\circ} 5 \ldots, \$ 23$.

40. Ainsi, la Cour suprême canadienne, dans l'affaire Febles c. Canada (Citoyenneté et Immigration), 2014 CSC 68 du 30 octobre 2014, a considéré que tout individu soupçonné d'avoir commis un crime grave de droit commun était automatiquement exclu, même s'il avait purgé sa peine et avait depuis renoncé à toute activité criminelle.

41. Voir HCR, Principes directeurs sur la protection internationale $n^{\circ} 5 \ldots, \$ 22$ et Note d'information sur l'application des clauses d'exclusion $\ldots . ., \$ 64-71$ ainsi que M. Tissier-Raffin, La qualité de réfugié de l'article 1 de la Convention de Genève..., p. 161-162.

42. Cette approche dite globale est notamment prônée et explicitée par le HCR dans ses Principes directeurs sur la protection internationale $n^{\circ} 5$..., $\$ 31$ et Note d'information sur l'application des clauses d'exclusion..., $\$ 99-100$.

43. Sur cet aspect, voir la conclusion de la présente étude.

44. Ces garanties procédurales sont notamment évoquées dans la Note d'information sur l'application des clauses d'exclusion..., $\$ 98$ sq. et dans la directive 2013/32/UE du Parlement européen et du Conseil du 26 juin 2013 relative à des procédures communes pour l'octroi et le retrait de la protection internationale. Voir C. Pouly, «Les garanties procédurales dans le nouveau régime d'asile européen commun », L'actualité juridique. Droit administratif, $\mathrm{n}^{\circ}$ 41, décembre 2013, p. 2358 sq.

45. La CNDA a ainsi pu souligner dans une décision $n^{\circ} 13024407$ du 27 février 2015 que «l'office comme le juge de l'asile ont la faculté, sous réserve du respect des droits de la défense et de la procédure contradictoire, d'opposer à tout stade de la procédure cette clause d'exclusion à toute personne dont les craintes de persécutions dans leur pays d'origine ont été préalablement jugées fondées, y compris dans le cadre d'une contestation d'une décision de retrait pour fraude» (nous soulignons).

46. Pour une appréciation de ce principe dans le cas français, voir C. Teitgen-Colly, «Réfugiés et protection subsidiaire», in Dictionnaire permanent Droit des étrangers, Montrouge, Éditions législatives, $\mathrm{n}^{\circ} 102$. 
parcours personnel, cette préoccupation est d'autant plus grande s'agissant d'individus soupçonnés d'avoir participé à la commission de crimes graves, dont il faut établir la réalité, ainsi que les circonstances précises de l'implication de l'intéressé. Ainsi, l'exigence d'un examen individualisé paraît d'autant plus fondamentale que l'on se trouve face à un possible cas de mise en œuvre de la clause d'exclusion. L'effectivité du principe de l'instruction individualisée peut toutefois être mise à mal dans les hypothèses d'afflux massif de demandeurs d'asile. Dans ces circonstances, les pays d'accueil les plus sollicités peuvent procéder à des reconnaissances collectives du statut de réfugié sur une base prima $\mathrm{facie}^{47}$. En effet, joue alors une présomption d'éligibilité au statut en faveur des membres d'une population clairement identifiée comme ayant légitimement fui une situation dangereuse. Or, ce sont précisément dans ces importants contingents de demandeurs d'asile fuyant au même moment un conflit armé par exemple que peuvent potentiellement se trouver des personnes ayant participé à des exactions. Pour éviter un dévoiement du système, le HCR recommande alors que la présomption d'éligibilité ne s'applique pas aux combattants actifs; les demandes présentées par ces individus devront nécessairement faire l'objet d'une instruction individualisée, afin que soit établie ou au contraire écartée leur implication dans la commission de certains crimes ${ }^{48}$. Cette solution suppose également que les clauses d'exclusion ne soient jamais appliquées collectivement, malgré la complexité du contexte dans lesquelles les demandes sont présentées ${ }^{49}$.

Un autre principe, dont l'importance doit être constamment rappelée, est celui de la confidentialité devant gouverner l'instruction de toute demande d'asile ${ }^{50}$, y compris lorsqu'est en jeu la clause d'exclusion. Il est cependant admis que, «dans des circonstances exceptionnelles, un contact avec le pays d'origine peut être justifié pour des raisons de sécurité nationale», sous réserve que l'existence de la demande d'asile ne soit pas révélée ${ }^{51}$. Cette position se comprend aisément s'agissant d'un individu dont il est admis qu'il peut craindre des persécutions dans son État d'origine, même si ses actes - dont la réalité doit être démontrée par exemple à partir des informations obtenues de la part des autorités du pays d'origine - peuvent par ailleurs conduire au refus d'une protection conventionnelle ou subsidiaire.
Il convient d'ailleurs de souligner que, dans un souci de protection de l'intégrité du système de l'asile, le principe de confidentialité continue à jouer même lorsqu'une décision finale d'exclusion a été rendue ${ }^{52}$.

\section{Les difficultés d'application du principe pourtant essentiel du contradictoire}

Le principe du contradictoire est affirmé tant en droit interne, qu'en droit international, au titre des principes généraux du droit irriguant toute procédure contentieuse digne d'un État de droit ${ }^{53}$. Applicable à toute procédure d'examen d'une demande d'asile, il revêt une importance singulière dans le contentieux de l'exclusion du fait de la gravité des actes reprochés au demandeur et de leurs conséquences : aussi l'intéressé doit-il être mis en mesure de répondre aux éventuels soupçons de participation à un crime international ou de tout autre acte visé à l'article 1.F de la Convention de Genève. Si le fondement et le sens du principe sont clairs, la mise en œuvre de la règle du contradictoire peut ponctuellement être rendue difficile, par exemple en cas d'absence du requérant (ou d'un interprète s'il ne maîtrise pas la langue du pays d'accueil) à l'audience devant les instances d'instruction ou à défaut de représentation par un avocat pour défendre convenablement sa position ${ }^{54}$. Il existe également des jurisprudences sanctionnant les instances de l'asile n'ayant pas notifié à l'intéressé, ou ne l'ayant pas fait dans un délai raisonnable, l'invocation de la clause d'exclusion à son encontre: une telle pratique contredit le principe du contradictoire et plus largement l'équité de la procédure puisque le demandeur n'est donc pas mis en mesure de s'expliquer sur les actes qui lui sont reprochés et qui justifient son exclusion du statut de réfugié ou de la protection subsidiaire ${ }^{55}$.

C'est également la logique du contradictoire qui s'oppose à l'admission de preuves confidentielles pour fonder une décision d'exclusion : selon le HCR, des preuves anonymes peuvent exceptionnellement être admises (par exemple pour protéger la sécurité des témoins) si «la capacité du demandeur d'asile à contester le contenu de la preuve n'est pas trop entravée», dans le respect de son droit «à un procès équitable ${ }^{56}$. Le lien se crée ici tout naturellement entre le nécessaire respect de normes

47. HCR, Guide des procédures et critères..., $\$ 44$.

48. HCR, Principes directeurs sur l'application des clauses d'exclusion de l'article 1.F de la Convention de 1951 relative au statut des réfugiés dans les situations d'afflux massif, février 2006, $\$ 9$ sq. Ce document expose également d'utiles principes opérationnels pour la gestion de ce contentieux particulier. 49. Ibid., $\$ 18$.

50. Principe affirmé par le HCR mais aussi les textes européens (art. 48 de la directive 2013/32/UE) et les jurisprudences nationales (voir notamment, pour la France, la décision de la Commission de recours des réfugiés du $1^{\text {er }}$ juin 2007, M. O., nº 05035520/561440 ou celle du Conseil d’État du $1^{\text {er }}$ octobre 2014, M. Erden, $\mathrm{n}^{\circ} 349560$ ).

51. HCR, Principes directeurs sur la protection internationale $n^{\circ} 5 \ldots, \S 33$.

52. HCR, Note d'information sur l'application des clauses d'exclusion $\ldots, \$ 104$

53. Pour le cas français, voir notamment CE, 28 février 1996, $\mathrm{n}^{\circ} 145062 ; \mathrm{CE}, 1^{\text {er }}$ octobre $2015, \mathrm{n}^{\circ} 349560$; et une étude des implications du principe du contradictoire et d'autres garanties procédurales en droit français par J. Krulic, «La réforme de la procédure devant la Cour nationale du droit d'asile», L'actualité juridique. Droit administratif, n 41, décembre 2013, p. 2371 sq.

54. Ces difficultés, non spécifiques au contentieux de l'exclusion, sont évoquées dans les articles précités parus dans L'actualité juridique. Droit administratif.

55. Voir par exemple Cour fédérale du Canada, Maloufc. Canada (ministre de la Citoyenneté et de l'Immigration), 31 octobre 1994, nº IMM-2186-94 et CE, 14 mars 2011, M. A., nº 329909.

56. HCR, Principes directeurs sur la protection internationale $n^{\circ} 5 \ldots, \S 36$ 
procédurales élémentaires telles que le contradictoire et la question de la preuve de l'implication d'un individu dans la commission d'actes justifiant son exclusion.

\section{B. Un standard de preuve élevé mais parfois mal respecté}

L'étude de la preuve dans le contentieux de l'exclusion suppose de commencer par se demander sur qui pèse cette charge et avec quelle intensité (1), avant d'aborder les moyens qui peuvent être utilement présentés (2), en gardant à l'esprit le principe fondamental - prôné par le HCR - selon lequel le doute doit toujours bénéficier au demandeur d'asile ${ }^{57}$.

\section{Des appréhensions variables de la charge et du niveau de preuve requis en matière d'exclusion}

Du fait de la nature exceptionnelle des clauses d'exclusion et du principe général de droit selon lequel la charge de la preuve incombe au demandeur, c'est aux autorités compétentes de l'État d'accueil de prouver la réalité des actes permettant d'exclure un demandeur de la protection internationale ou subsidiaire ${ }^{58}$. Malgré la clarté de cette règle, la doctrine constate des interprétations nationales variables de l'article 1.F de la Convention de Genève évoquant des «raisons sérieuses de penser» qu'un candidat à l'asile est impliqué dans la commission d'un crime pouvant justifier une exclusion ${ }^{59}$. Tout au plus peut-on souligner l'existence d'un consensus autour de l'idée que cette formule suppose un niveau de preuve moins élevé que celui - typique des systèmes anglo-saxons et privilégié par les juridictions pénales internationales - de l'établissement de la culpabilité «au-delà de tout doute raisonnable ${ }^{6}{ }^{\circ}$. Si les juridictions internes en tirent des conséquences variables, certains auteurs considèrent que joue en réalité une véritable présomption de l'implication du demandeur d'asile dans les faits litigieux, présomption pouvant toutefois être combattue par la preuve contraire ${ }^{61}$.
Devant ces difficultés, il est primordial d'insister sur le caractère insuffisant de l'un ou l'autre des éléments suivants, à lui seul, pour opposer valablement une clause d'exclusion: la mise en accusation par un tribunal étranger du candidat à l'asile ou l'enclenchement d'une procédure d'extradition contre lui ${ }^{62}$, l'existence d'un contexte génocidaire ou d'une situation d'attaque généralisée ou systématique de la population civile dans son pays d'origine $^{63}$ ou encore son inscription sur une liste de membres présumés d'organisations terroristes ${ }^{64}$. Cette dernière solution, adoptée par des juridictions suprêmes et la Cour de justice de l'Union européenne, est opportunément venue encadrer une pratique excluant par trop facilement des individus ayant été associés à des réseaux terroristes. Au-delà de cet exemple, selon le HCR et la jurisprudence majoritaire, de simples suspicions ne sont donc pas suffisantes pour activer le mécanisme de l'exclusion.

\section{La nécessité de réunir des éléments de preuve objectifs contre le demandeur pour l'exclure}

En cas de soupçon de la participation d'un demandeur d'asile à la commission d'un crime justifiant une exclusion, la seule logique conforme à l'objectif protecteur de la Convention de Genève est de réunir un faisceau d'indices objectifs permettant d'établir son implication personnelle. Autrement dit, il s'agit d'apporter des preuves claires et crédibles de la participation de l'intéressé à la commission des actes litigieux. Parmi les éléments pertinents, est fréquemment mise en avant la mise en accusation de l'intéressé devant une juridiction pénale internationale, ou mieux, sa condamnation définitive par ce juge international. Cette solution se justifie par le niveau d'exigence caractérisant l'enclenchement d'une telle procédure, présumé plus élevé que celui d'une procédure classique devant le juge interne ${ }^{65}$. A contrario, l'acquittement d'un candidat à l'asile par une juridiction pénale internationale peut mettre l'État d'accueil dans une situation difficile: si la culpabilité de l'intéressé n'a pas été établie, il peut en effet subsister un doute quant à l'absence totale de lien entre lui et le

57. HCR, Guide des procédures et critères..., \$196 et 203-204.

58. HCR, Note d'information sur l'application des clauses d'exclusion..., $\$ 105$.

59. Voir M. Holvoet, «Harmonizing Exclusion under the Refugee Convention by Reference to the Evidentiary Standards of International Criminal Law ", Journal of International Criminal Justice, vol. 12, nº 5, 2014, p. 1041 sq.; J.-Y. Carlier, P. D’Huart, "L'exclusion du statut de réfugié: cadre général», in Asile et extradition..., p. 6-9.

60. Ibid. et M. Tissier-Raffin, La qualité de réfugié de l'article 1 de la Convention de Genève..., p. 174 sq.

61. Dans le cas français, voir C. Teitgen-Colly, «Réfugiés et protection subsidiaire», $\mathrm{n}^{\circ} 233$, citant par exemple CE, 17 janvier $2011, \mathrm{n}^{\circ} 316678$.

62. Dans la décision précitée du 27 février 2015, la CNDA souligne ainsi que «Les seuls éléments de présomption portés à la connaissance de la cour et versés au débat contradictoire sur lesquels repose la demande d'extradition, comportant un faisceau de témoignages de tiers et d'écoutes téléphoniques, s'ils justifient pleinement l'intérêt pour les autorités turques de les confronter aux déclarations de l'intéressé, n'apparaissent pas en l'état suffisants pour établir le constat qu'il existe des raisons sérieuses de penser qu'une responsabilité individuelle dans l'accomplissement d'un crime grave de droit commun peut être imputée au requérant».

63. Voir illustrations dans C. Bauloz, «L'apport du droit international pénal...», p. 49 sq.

64. Ainsi, dans l'affaire Ezokola c. Canada (Citoyenneté et Immigration) du 19 juillet 2013, CSC 40 n $^{\circ} 34470$, la Cour suprême du Canada rejoint-elle la position de la CJUE dans l'affaire Bundesrepublik Deutschland c. B (C-57/o9) et D (C-101/og), 9 novembre 2010, selon laquelle le fait, pour une personne, d'avoir appartenu à une organisation inscrite sur une liste antiterroriste ne constitue pas automatiquement une raison sérieuse de penser que cette personne a commis un crime pouvant entraîner une exclusion. Voir aussi CNDA, 15 juillet 2014, M. S., n 11016153, sur le caractère non suffisant de la seule appartenance d'un requérant à une organisation notoirement connue comme s'étant rendue coupable d'actes terroristes (Tigres libérateurs de l'Eelam tamoul - LTTE).

65. HCR, Note d'information sur l'application des clauses d'exclusion..., $\$ 107$. 
crime poursuivi par le juge international, ou d'autres actes commis dans le même contexte criminel ${ }^{66}$. Dans ce type d'hypothèse, il convient de faire jouer le principe précité selon lequel le doute doit bénéficier au candidat à l'asile.

Autre élément objectif pouvant être valablement retenu pour enclencher la clause d'exclusion contre un demandeur d'asile, son statut de membre d'un gouvernement ou d'un autre groupement notoirement impliqué dans la commission de crimes internationaux, et qui ne s'en désolidarise pas alors qu'il avait la possibilité de le faire ou qu'il avait la capacité d'influencer de façon significative les activités en cause ${ }^{67}$. En dehors des individus occupant de tels postes à responsabilités, il convient d'examiner

[...] le rôle qu'a effectivement joué la personne concernée dans la perpétration des actes [criminels], sa position au sein de l'organisation, le degré de connaissance qu'elle avait ou était censée avoir des activités de celle-ci, les éventuelles pressions auxquelles elle aurait été soumise ou d'autres facteurs susceptibles d'influencer son comportement ${ }^{68}$.

Cette appréciation du rôle personnel joué par le candidat à l'asile peut notamment être faite à partir de plusieurs sources d'informations, publiques et aisément accessibles, en particulier des rapports d'organisations non gouvernementales (ONG) ou d'organes onusiens ${ }^{69}$.

En conclusion, la présente étude ne visait pas à suggérer une transposition au contentieux de l'exclusion de l'ensemble des garanties d'un procès pénal équitable, solution qui ne serait ni correcte juridiquement ${ }^{70}$, ni réalisable d'un point de vue pratique. Son ambition était simplement de souligner la nécessité de respecter certains principes fondamentaux tenant au principe de légalité et aux garanties de procédure les plus élémentaires, afin d'assurer un minimum de droits au demandeur d'asile à qui la clause d'exclusion, aux effets aussi graves que définitifs, pourrait être opposée. Ce souci de préserver les droits fondamentaux minimums de l'intéressé doit également être gardé à l'esprit après l'adoption de la décision l'excluant finalement du statut de réfugié ou de la protection subsidiaire. En effet, cet exclu ne peut être renvoyé vers son pays d'origine dès lors qu'est établie l'existence d'un risque avéré d'atteinte à sa vie ou à son intégrité ${ }^{71}$. Il se trouve alors placé dans une situation paradoxale puisqu'il ne peut pas obtenir de statut dans le pays d'accueil, ni être renvoyé vers son État d'origine $^{72}$, ce qui interroge sur la qualité de ses conditions d'existence et l'effectivité de ses droits fondamentaux. Or, la détermination d'un statut international ou même de politiques nationales claires à l'égard des exclus est encore loin d'être faite, ni même véritablement pensée ${ }^{73}$. Par conséquent et en tenant aussi compte du risque de fragmentation du droit des réfugiés précédemment évoqué, on pourrait considérer avec certains auteurs que la clause d'exclusion pose finalement plus de problèmes qu'elle n'en résout et qu'elle devrait être abandonnée. Il faudrait alors lui substituer un système dans lequel les individus « exclus potentiels» recevraient une protection internationale, mais feraient en contrepartie, et de façon certaine, l'objet de poursuites pénales pour les crimes à la commission desquels ils ont participé: il s'agirait donc «de ne plus sanctionner l'indigne par simple abstention, l'exclusion du statut de réfugié, mais par une action, l'action judiciaire et le jugement $»^{74}$. Loin d'être consensuelle, cette proposition d'évolution présenterait à tout le moins l'avantage de placer ce type de candidat à l'asile débouté face à une procédure juridictionnelle dont on peut espérer qu'elle serait plus respectueuse des droits fondamentaux que les actuelles procédures d'examen menant à une décision d'exclusion.

66. J. Rikhof, The Criminal Refugee..., p. 114 .

67. HCR, Note d'information sur l'application des clauses d'exclusion..., $\$ 58 \mathrm{sq}$.

68. CJUE, 9 novembre 2010, Bundesrepublik Deutschland, $\$ 97$ ou, pour une application en droit français: CNDA, 15 juillet 2014, M. S.

69. Suivant en cela la méthodologie adoptée au sein de la Cour pénale internationale: voir M. Holvoet, « Harmonizing Exclusion... », p. 1054. Pour un exemple de prise en compte explicite de rapports d'ONG en droit français, voir CNDA, 18 novembre 2014, M. K., $\mathrm{n}^{\circ} 090118932$.

70. Il convient de rappeler que, selon des jurisprudences européennes et nationales concordantes, l'article 6 de la Convention européenne des droits de l'homme sur le droit au procès équitable n'est pas applicable aux procédures d'octroi de l'asile.

71. Solution découlant non du droit des réfugiés mais du droit international des droits de l'homme, en particulier de l'article 3 de la Convention européenne des droits de l'homme, refusant l'éloignement d'un individu vers un pays où il risque la mort ou de subir des actes de torture ou des traitements inhumains ou dégradants. Ce principe est notamment repris à l'article 21, $\$ 1$, de la directive "Qualification».

72. Voir J. Matringe, «La situation de l'exclu au regard de l'extradition», in Asile et extradition..., p. 257-297.

73. Pour une illustration dans le cas néerlandais, voir J. Reijven, J. Van Wijk, «Caught in Limbo: How Alleged Perpetrators of International Crimes Who Applied for Asylum in the Netherlands Are Affected by a Fundamental System Error in International Law », International Journal of Refugee Law, vol. 26, n 2 2, 2014, p. 248-271.

74. J.-Y. Carlier, P. D’Huart, «L'exclusion du statut de réfugié... », p. 24 sq. 